\title{
Classification of long Gamma Ray Bursts using cosmologically corrected temporal estimators
}

\author{
Nicolas A. Vasquez ${ }^{1}$ and Christian Vasconez ${ }^{2}$ \\ ${ }^{1}$ Observatorio Astronomico de Quito \\ Av Gran Colombia S/N, Parque de la Alameda, Quito, Ecuador \\ email: nvasquez.observatorio@epn.edu.ec \\ ${ }^{2}$ Observatorio Astronomico de Quito \\ Av Gran Colombia S/N, Parque de la Alameda, Quito \\ email: c.vasconez@epn.edu.ec
}

\begin{abstract}
The canonical classification of GRBs establishes two types of bursts, long and short. Although an intermediate class of GRBs was suggested, its existence is not yet conclusive. In the present work, we explore the temporal classification of GRBs in the burst frame, because in recent years the statistics of bursts with known redshifts has increased. We studied a sample of Swift GRBs with known redshifts to determine three different time estimators: autocorrelation functions, emission times and duration times. In order to look for a subclass in long GRBs, we studied the distribution of the cosmologically corrected time estimators. The distribution of time estimators of the sample suggests an internal division of long GRBs. The proposed bimodality is also supported in the isotropic luminosity - time estimator planes and we discuss some possible implications of the classification of GRBs in the burst frame.
\end{abstract}

Keywords. gamma rays: bursts, gamma rays: observations

\section{Introduction}

The introduction of a third class of bursts (Horvath et al. (2010)) within the canonical classification of GRBs (Kouveliotou et al. (1993)) has improved our understanding of the progenitors and radiation mechanisms of these explosions. Nevertheless, classifications of bursts has been done in the observer frame which includes some of the underlying effects of the cosmological expansion of the Universe. In the present study we performed a temporal classification in the burst frame as a extension of a previous work (Vasquez \& Kawai (2010)).

\section{Data analysis}

Our sample is composed of 15 GRBs detected simultaneously by the Swift and Suzaku missions with measured redshifts. We compute the duration time of the 50 percent of the total fluence $\left(\mathrm{T}_{50}\right)$, the emission time of the 50 percent of the total fluence $\left(\mathrm{t}_{50}\right.$; Mitrofanov et al. (1998)), and the autocorrelation function (ACF) of the light curve evaluated at FWHM (Link et al. (1995)) based on Monte Carlo simulations of the light curves. We found that the emission time is a better temporal estimator as is shown in Figure 1. The correlation coefficient between the isotropic luminosity improves from -0.28 to 0.78 when the four long-dim bursts are taken as different type of GRBs. This behavior is recurrent with the other temporal estimators. 


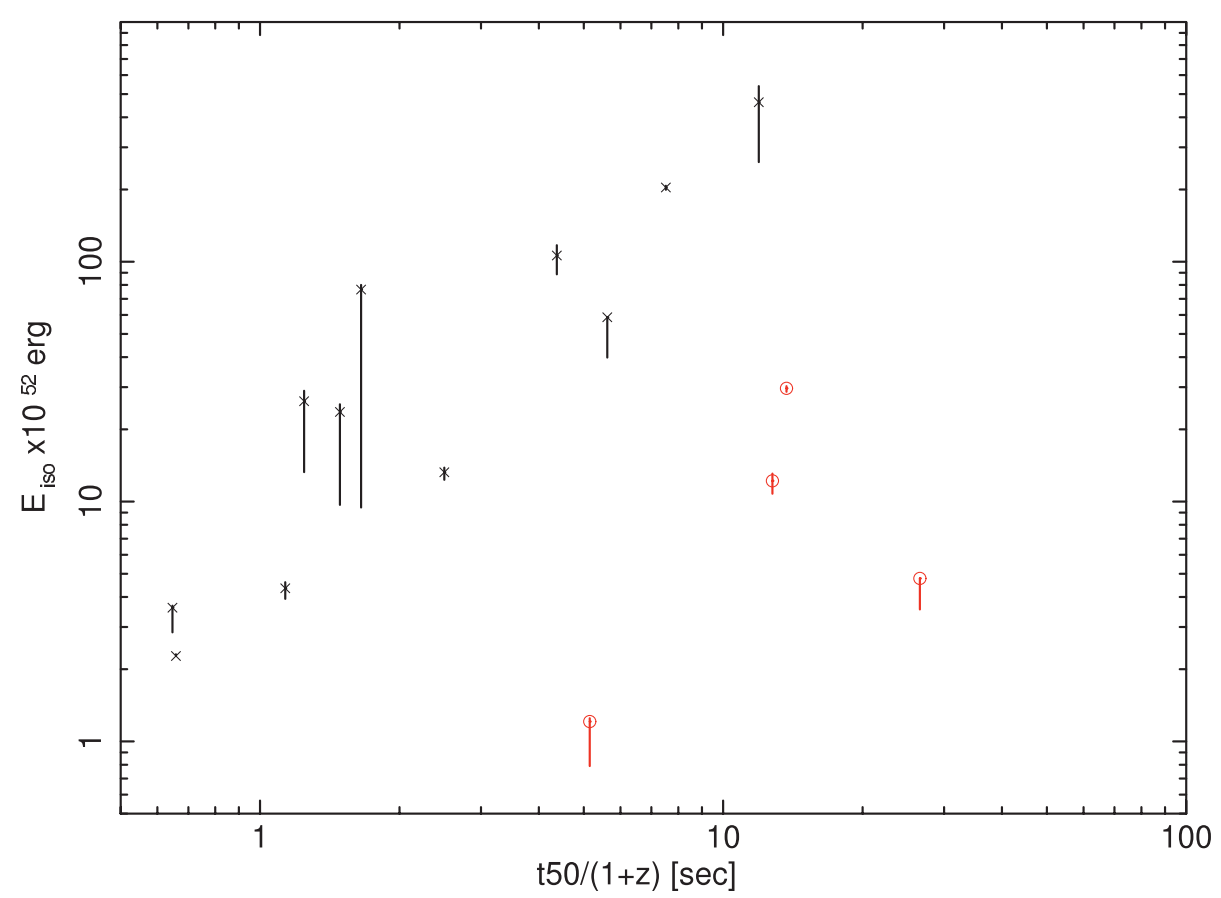

Figure 1. Isotropic luminosity as a function of the emission time $\left(t_{50}\right)$ in the $50-150 \mathrm{keV}$ band. In the right bottom corner, the four long-dim bursts are localized.

\section{Implications}

One of the most intriguing questions is to know if the classifications of bursts in the observer frame are hiding some intrinsic properties due to cosmological effects. Borgonovo et al. (2007) show that long GRBs have a bimodal distribution when the ACF is cosmologically corrected (Borgonovo et al. (2007)). Following this idea, we also found a similar bimodal distribution of long GRBs when the ACF is computed in the rest frame. Since we expect that cosmological dilation effects are the same for all timescales, we also extend the bimodality for other time estimators: duration times of outburst activity $\left(\mathrm{T}_{50}\right)$ and the time intervals where the engine is in the most active phase $\left(t_{50}\right)$. There are four GRBs, that in the time-distance plane, form a subgroup. Long-near bursts and long-distant bursts, seem to have different origin and behavior. In the light curves, we also observed that there are some common trends: the time history is composed of broad pulses that underlie a longer active phase. Evidence for a subclass of GRBs is also present in the time-energy planes, which reinforces the possibility of different engines or progenitor for GRBs that have longer active phases, are dimmer and have smaller redshifts.

\section{References}

Borgonovo L., et al. 2007, A\& $A, 465,765$

Horvath Z., et al. 2010, ApJ, 713, 552

Kouveliotou C., et al. 1993, ApJL, 413, 101

Link, B., Epstein, R. I., \& Priedhorsky, W. C. 1995, ApJ, 631, 976

Mitrofanov I., et al. 1998, ApJ, 522, 1069

Vasquez, N. \& Kawai, N. 2010, Inter. J. of Modern Physics D, 19, 997 